\title{
Defeito do anel fibroso mitral posterior com aneurisma de átrio esquerdo e insuficiência mitral: tratamento cirúrgico com sucesso
}

\author{
Ronaldo Machado BUENO*, Mário CERDEIRA Jr. ${ }^{*}$, Henry ABENSUR* ${ }^{*}$ Amílton SILVA Jr. ${ }^{*}$, \\ Osmar Araújo CALIL*, Vicente ÁVILA NETO*, Ricardo F.A. MELO*,
}

RBCCV 44205-478

\begin{abstract}
Bueno R M, Cerdeira Jr. M, Abensur H, Silva Jr. A, Calil O A, Ávila Neto V, Melo R F A-Defeito do anel fibroso mitral posterior com aneurisma de átrio esquerdo e insuficiência mitral: tratamento cirúrgico com sucesso. Rev Bras Cir Cardiovasc 1999; 14 (4): 348-54.
\end{abstract}

\begin{abstract}
RESUMO: Aneurismas em átrio esquerdo são pouco comuns, podendo ocorrer na aurícula ou na parede do átrio esquerdo. Freqüentemente, são assintomáticos, podendo ocorrer arritmias, fenômenos tromboembólicos ou insuficiência cardíaca como complicação da sua evolução. Apresentamos paciente de 39 anos, do sexo feminino, com defeito do anel posterior da valva mitral levando a grande dilatação aneurismática da parede posterior do átrio esquerdo com insuficiência mitral. O diagnóstico foi feito pela radiografia de tórax (abaulamento de silhueta cardíaca esquerda) e ecocardiograma (grande aneurisma do átrio esquerdo posteriormente à parede posterior do ventrículo esquerdo com insuficiência mitral). O estudo hemodinâmico sugeriu pseudo-aneurisma de ventrículo esquerdo. Submetida a tratamento cirúrgico com auxílio da circulação extracorpórea, realizou-se anuloplastia mitral e exclusão do aneurisma com reconstrução do assoalho do átrio esquerdo com retalho de pericárdio bovino. A paciente apresentou boa evolução pós-operatória, recebendo alta hospitalar no oitavo dia em boas condições clínicas.
\end{abstract}

DESCRITORES: Aneurisma cardíaco, congênito, cirurgia. Insuficiência da valva mitral, cirurgia. Átrio. Valva mitral, cirurgia.

\section{INTRODUÇÃO}

Os aneurismas do átrio esquerdo são raros, tendo sido descritos em 1938 por Semans e Taussig (1). São de etiologia congênita ou adquirida, podendo acometer a aurícula esquerda ou, menos freqüentemente, a parede do átrio esquerdo (2). A presença de aneurisma em átrio direito é ainda mais rara (3). Freqüentemente, esses aneurismas são assintomáticos, mas podem apresentar, como complicações, sinais de insuficiência cardíaca, arritmias ou embolias sistêmicas. A possibilidade de ocorrência de tais complicações faz com que a intervenção cirúrgica seja o tratamento de escolha (4-6).

Relatamos o caso de paciente com defeito no anel fibroso mitral posterior, com aneurisma em parede posterior do átrio esquerdo e insuficiência mitral, submetido a tratamento cirúrgico com sucesso.

\section{RELATO DE CASO}

Paciente do sexo feminino, 39 anos de idade,

Trabalho realizado na Real e Benemérita Sociedade Portuguesa de Beneficência. São Paulo, SP, Brasil.

Recebido para publicação em abril de 1999

* Do Serviço do Dr. Ricardo Fernandes de Azevedo Melo.

Endereço para correspondência: Ronaldo Machado Bueno. Rua Loureiro da Cruz, 121 apt. 22. Aclimação. São Paulo, SP,Brasil. Cep 01529-020.

Tel: (011) 3277-5665.e-mail: galaxia@dialdata.com.br 
Bueno R M, Cerdeira Jr. M, Abensur H, Silva Jr. A, Calil O A, Ávila Neto V, Melo R F A - Defeito do anel fibroso mitral posterior com aneurisma de átrio esquerdo e insuficiência mitral: tratamento cirúrgico com sucesso. Rev Bras Cir Cardiovasc1999; 14 (4): 348-54

sem antecedentes reumáticos, com história de dispnéia desde os 24 anos, inicialmente aos grandes esforços, evoluindo gradativamente para médios esforços. Neste último ano, episódios de palpitação, mal estar, sudorese e dispnéia relacionados à presença de taquicardia paroxística supraventricular ao eletrocardiograma, necessitando internações hospitalares para controle. Em dezembro de 1998, durante internação para tratamento cirúrgico, apresentou episódios de síncope, com confusão mental temporária. Propedêutica neurológica não evidenciou alterações significativas, apresentando boa evolução, sem qualquer seqüela.

Ao exame clínico cardiológico apresentava, à ausculta cardíaca, sopro sistólico em foco mitral de intensidade III/VI (de Levine), com irradiação para axila esquerda. A radiografia de tórax demonstrou aumento da área cardíaca com abaulamento de siIhueta cardíaca esquerda (Figura 1). Ao eletrocardiograma, presença de ritmo sinusal, sem arritmias, com desvio de eixo do QRS para a direita.

Realizado ecocardiograma transtorácico que mostrou a presença de importante insuficiência mitral e aumento assimétrico do átrio esquerdo com dilatação (aneurisma) da parede posterior (Figura 2).

O estudo hemodinâmico evidenciou insuficiência mitral importante com presença de dilatação aneurismática posteriormente ao ventrículo esquerdo, sugestiva de pseudo-aneurisma de ventrículo esquerdo; artérias coronárias sem anormalidades (Figura 3).

Manometria demonstrando hipertensão pulmonar moderada, com elevação da pressão capilar pulmonar (Tabela 1).

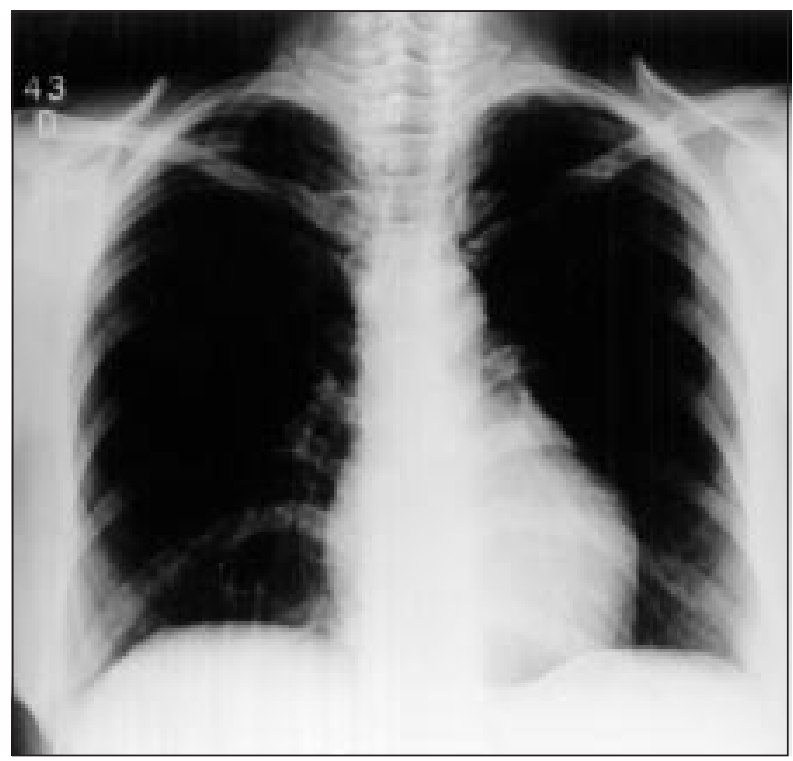

Fig. 1 - Radiografia de tórax em PA no pré-operatório mostrando abaulamento da silhueta cardíaca esquerda.

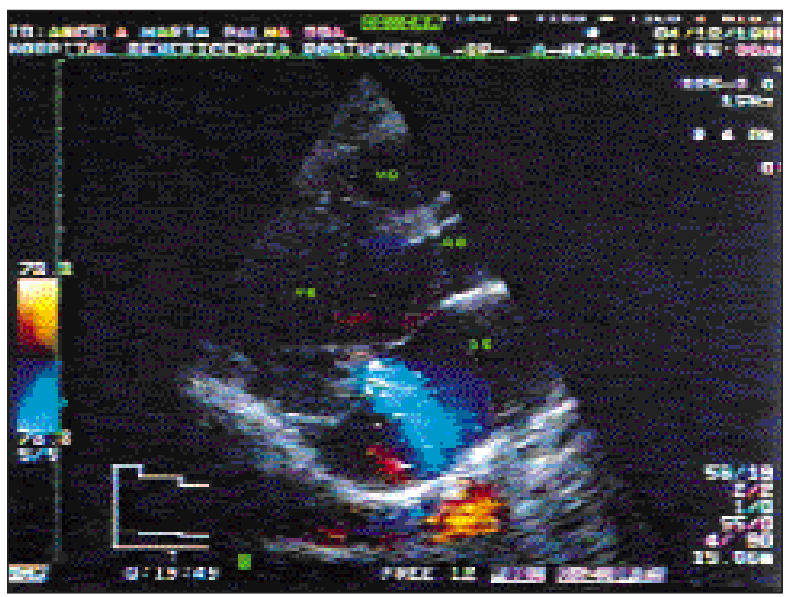

Fig. 2 - Ecocardiograma transtorácico. Corte paraesternal esquerdo longitudinal com mapeamento de fluxo em cores mostrando regurgitação mitral importante.

Submetida a tratamento cirúrgico em dezembro de 1998, foi realizado ecocardiograma transesofágico per-operatório que confirmou a presença de insuficiência mitral importante, com grande aneurisma da parede posterior do átrio esquerdo, crescendo posteriormente à parede posterior do ventrículo esquerdo, a qual se apresentava com boa contratilidade, sem evidência de pseudo-aneurisma (Figura 4). Realizada esternotomia mediana como via de acesso, notando-se grande dilatação em parede posterior do átrio esquerdo com crescimento em direção inferior sobre o sulco atrioventricular posterior e parede posterior do ventrículo esquerdo. As aurículas direita e esquerda não apresentavam alterações. Pericárdio íntegro. Abordagem cirúrgica por atriotomia esquerda, observando-se o anel fibroso mitral posterior mal definido, deformado e atrófico, sugerindo cúspide mitral posterior em contiguidade com

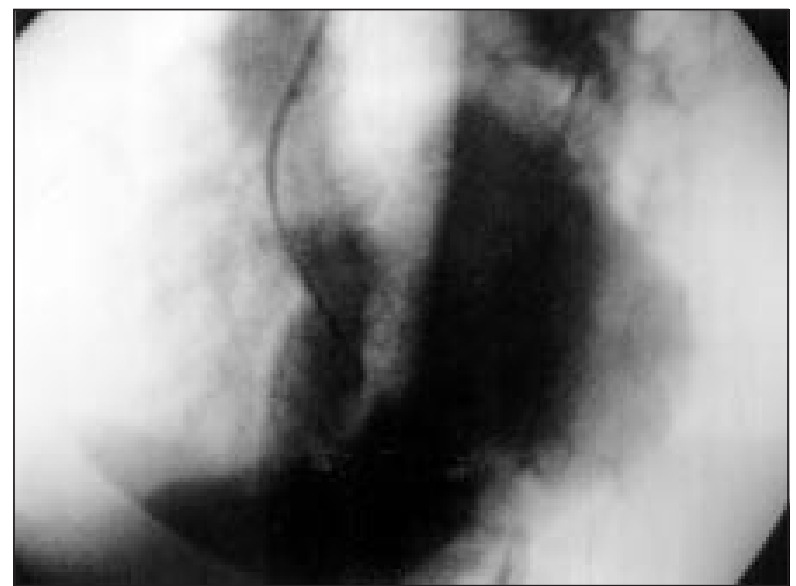

Fig. 3 - Ventriculografia esquerda evidenciando grande dilatação aneurismática posteriormente à parede posterior do ventrículo esquerdo sugerindo pseudo-aneurisma. 
Bueno R M, Cerdeira Jr. M, Abensur H, Silva Jr. A, Calil O A, Ávila Neto V, Melo R F A - Defeito do anel fibroso mitral posterior com aneurisma de átrio esquerdo e insuficiência mitral: tratamento cirúrgico com sucesso. Rev Bras Cir Cardiovasc1999; $14(4): 348-54$

TABELA 1

\begin{tabular}{ccccc}
\hline \multicolumn{5}{c}{ MANOMETRIA } \\
\hline \multirow{4}{*}{ LOCAL } & $\boldsymbol{S}$ & \multicolumn{4}{c}{ D1 } & D2 & $\boldsymbol{M}$ \\
\cline { 2 - 5 } & & & \\
\hline AD & ------ & --- & 20 \\
VD & 60 & 0 & 20 & ---- \\
TP & 60 & 25 & --- & 36 \\
CP & ---- & ---- & --- & 25 \\
VE & 120 & 0 & 28 & ---- \\
AO & 120 & 75 & ---- & 90 \\
\hline
\end{tabular}

$\mathrm{AD}$ = átrio direito; $\mathrm{VD}=$ ventrículo direito; $\mathrm{TP}$ = tronco pulmonar; $\mathrm{CP}=$ capilar pulmonar; $\mathrm{VE}=$ ventrículo esquerdo; $\mathrm{AO}=$ aorta; $\mathrm{S}$ = pressão sistólica; $\mathrm{D} 1$ = pressão diastólica inicial; D2= pressão diastólica final; $M=$ pressão média.

a musculatura atrial esquerda. Valva mitral sem calcificações, espessamentos ou fibrose importante das cúspides ou aparelho subvalvar. Cúspide mitral posterior sem sustentação, desabada, levando à insuficiência mitral importante. Presença de grande aneurisma em parede posterior do átrio esquerdo (assoalho), de parede delgada, friável, sem trombo em seu interior (Figura 5).

Realizada anuloplastia mitral (Figura 6) com reconstrução do assoalho do átrio esquerdo com retalho de pericárdio bovino tendo-se como limite, inferiormente, o próprio anel mitral posterior da anuloplastia, lateralmente, a desembocadura das veias pulmonares e, superiormente, a base da aurícula esquerda (Figura 7). O ecocardiograma transesofágico per-operatório, realizado logo após a correção cirúrgica, mostrou valva mitral suficiente, sem escape, e presença do retalho de pericárdio bovino

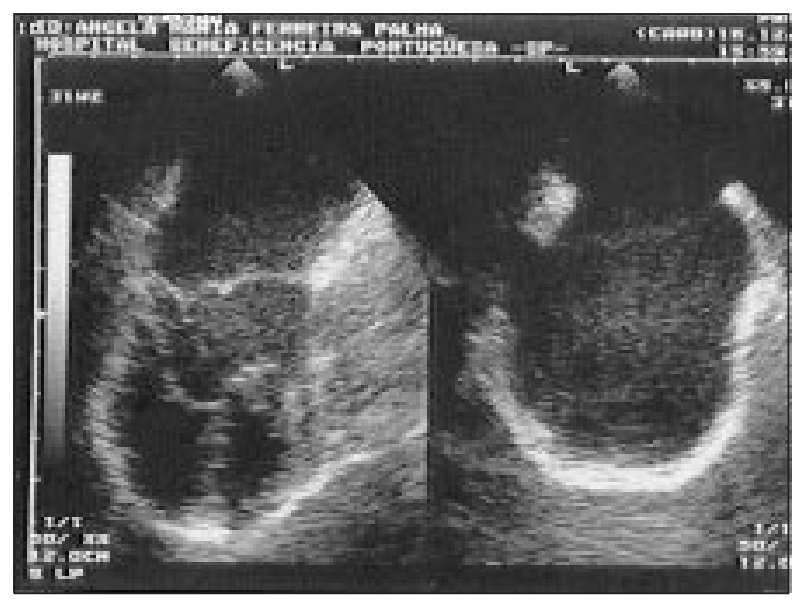

Fig. 4 - Ecocardiograma transesofágico. A: Corte longitudinal mostrando átrio esquerdo e ventrículo esquerdo. B: Corte longitudinal mostrando o imenso átrio esquerdo localizado posteriormente ao ventrículo esquerdo.

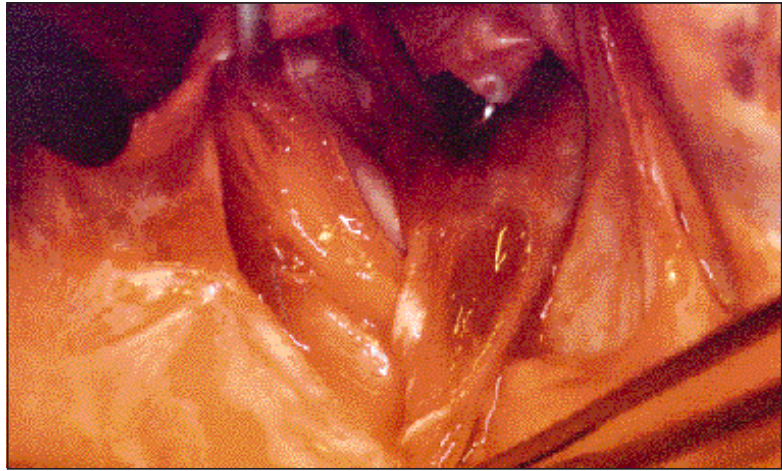

Fig. 5 - Visão cirúrgica do interior do átrio esquerdo. Notar a parede posterior do átrio esquerdo tracionada para fora da cavidade atrial esquerda (aneurisma da parede posterior do átrio esquerdo).

formando o novo assoalho do átrio esquerdo, com exclusão da região aneurismática (Figura 8 e 9). A radiografia de tórax no pós-operatório imediato demonstrou redução da área cardíaca, sem abaulamento da silhueta cardíaca esquerda (Figura 10) e o eletrocardiograma com ritmo sinusal e eixo de QRS normal. O pós-operatório transcorreu sem anormalidades, sem arritmias ou sinais de insuficiência cardíaca, tendo a paciente recebido alta hospitalar no oitavo dia de pós-operatório.

\section{COMENTÁRIOS}

Os aneurismas do átrio esquerdo não são lesões comuns. Quanto a etiologia podem ser congênitos (7) ou adquiridos (8). São considerados congênitos quando nenhuma doença cardíaca predisponente está presente. São adquiridos quando se desenvolvem secundariamente à doença reumática, tuberculose, miocardite sifilítica, trauma pós-substituição da valva mitral ou outras condições que evoluem com elevação importante da pressão atrial esquerda ou enfraquecimento do miocárdio (9-11).

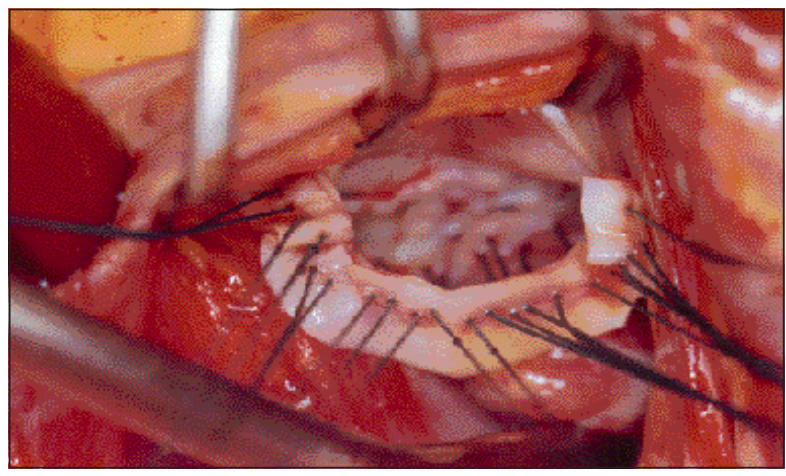

Fig. 6 - Visão cirúrgica. Anuloplastia mitral com retalho de pericárdio bovino. 
Bueno R M, Cerdeira Jr. M, Abensur H, Silva Jr. A, Calil O A, Ávila Neto V, Melo R F A - Defeito do anel fibroso mitral posterior com aneurisma de átrio esquerdo e insuficiência mitral: tratamento cirúrgico com sucesso.

Rev Bras Cir Cardiovasc1999. $14(4): 348-54$

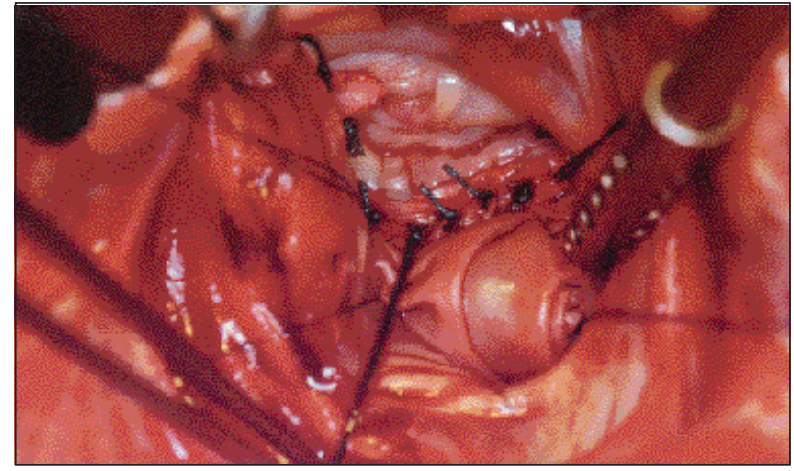

Fig. 7 - Visão cirúrgica do interior do átrio esquerdo. Notar retalho de pericárdio bovino formando novo assoalho (parede posterior) do átrio esquerdo, em continuidade com a anuloplastia mitral.

Estes aneurismas em átrio esquerdo são intra ou extrapericárdicos (12) podendo acometer a aurícula esquerda (13) ou, menos freqüentemente, o átrio esquerdo (14). Aneurismas da aurícula esquerda comumente se associam a defeitos do pericárdio (11). Outras lesões que podem estar associadas aos aneurismas de átrio esquerdo são: comunicação interatrial, drenagem anômala parcial das veias pulmonares e a presença de veia cava superior esquerda (15). A etiopatogenia ainda não está bem definida. Uma das possibilidades é que ocorra uma dilatação progressiva a partir de um ponto fraco na parede atrial. O exame histopatológico não demonstrou alteração estrutural importante da parede atrial nos casos estudados por RAVIKUMAR et al. (6) e GRINFELD et al. (7). No caso que relatamos, provavelmente, um defeito no anel mitral posterior em sua junção com o miocárdio da parede atrial esquerda determinou a formação de um ponto fraco com progressiva dilatação atrial levando, por sua relação com a valva mitral, à insuficiência valvar

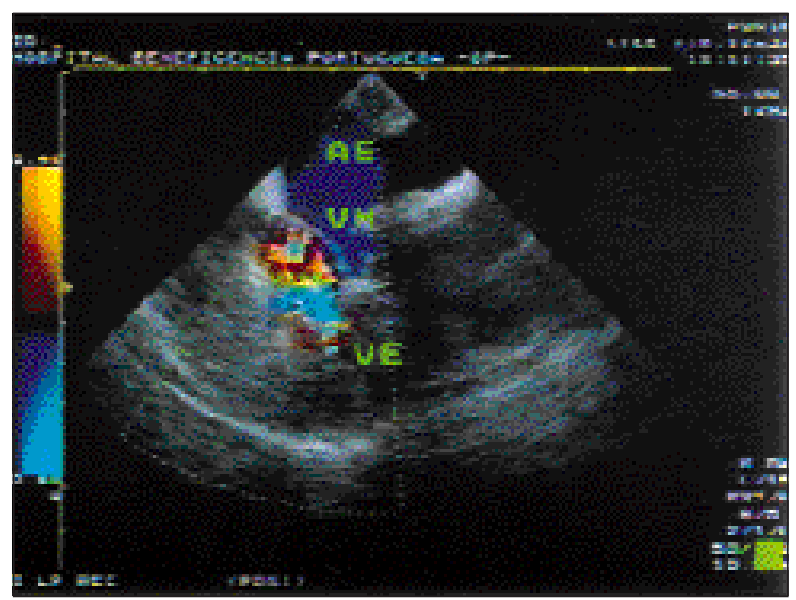

Fig. 8 - Ecocardiograma transesofágico intra-operatório. Corte transverso mostrando ausência de regurgitação mitral pósplastia valvar. mitral. O fato da paciente não apresentar história clínica, antecedentes, alterações ecocardiográficas ou laboratoriais, nem alterações anatômicas da valva mitral sugestivas de doença reumática, ou de outras doenças possíveis de levar à formação de aneurisma atrial esquerdo, sugere a etiologia congênita.

Clinicamente, a maioria desses pacientes é assintomática. Entretanto, sintomas como dispnéia, dor torácica, palpitação e síncope podem estar presentes $(6,7)$.

O eletrocardiograma é inespecífico, auxiliando no diagnóstico das arritmias (extra-sístoles supraventriculares, fibrilação atrial, taquicardia paroxística supraventricular) que podem estar associadas à doença (11).

A radiografia de tórax pode ser normal ou demonstrar abaulamento, com aumento da convexidade da borda cardíaca esquerda. Entretanto, nenhuma alteração radiológica é patognomônica da lesão $(2,16,17)$. O ecocardiograma transtorácico constitui excelente método diagnóstico na avaliação não-invasiva do átrio esquerdo, valva mitral e ventrículo esquerdo (18-20) Entretanto, aumento assimétrico do átrio esquerdo pode trazer certa dificuldade diagnóstica. No caso apresentado, houve crescimento assimétrico do átrio esquerdo com formação de dilatação aneurismática da parede posterior em direção inferior atrás da parede posterior do ventrículo esquerdo. Grandes aumentos atriais (átrio esquerdo gigante) ou aneurismas de átrio esquerdo podem causar o prolapso neste átrio posteriormente à parede posterior do ventrículo esquerdo $(8,21)$, com o diagnóstico errôneo de derrame pericárdico (22) bem como alterando a posição e contratilidade da parede póstero-basal do ventrículo esquerdo afetando sua função (21).

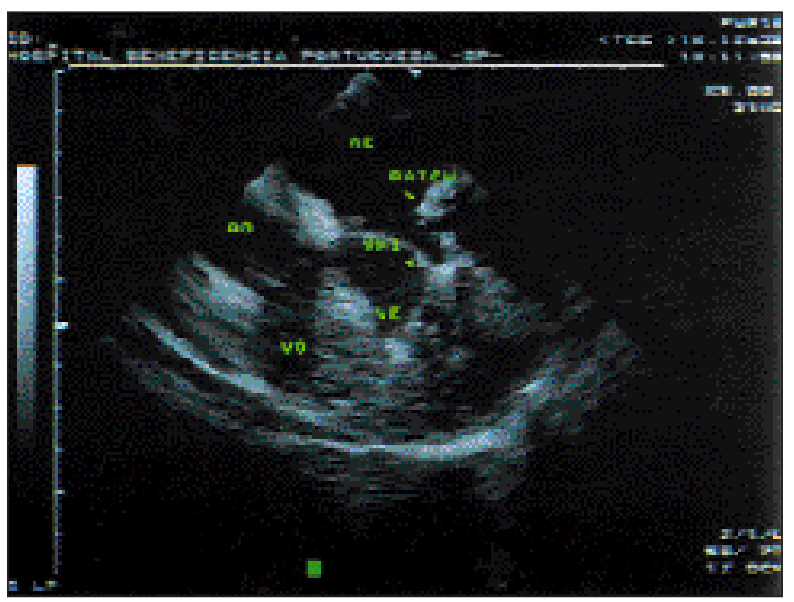

Fig. 9 - Ecocardiograma transesofágico intra-operatório. Corte transverso mostrando retalho de pericárdio bovino formando a nova parede posterior do átrio esquerdo, com exclusão da região aneurismática. 
Bueno R M, Cerdeira Jr. M, Abensur H, Silva Jr. A, Calil O A, Ávila Neto V, Melo R F A - Defeito do anel fibroso mitral posterior com aneurisma de átrio esquerdo e insuficiência mitral: tratamento cirúrgico com sucesso. Rev Bras Cir Cardiovasc1999; 14 (4): 348-54.

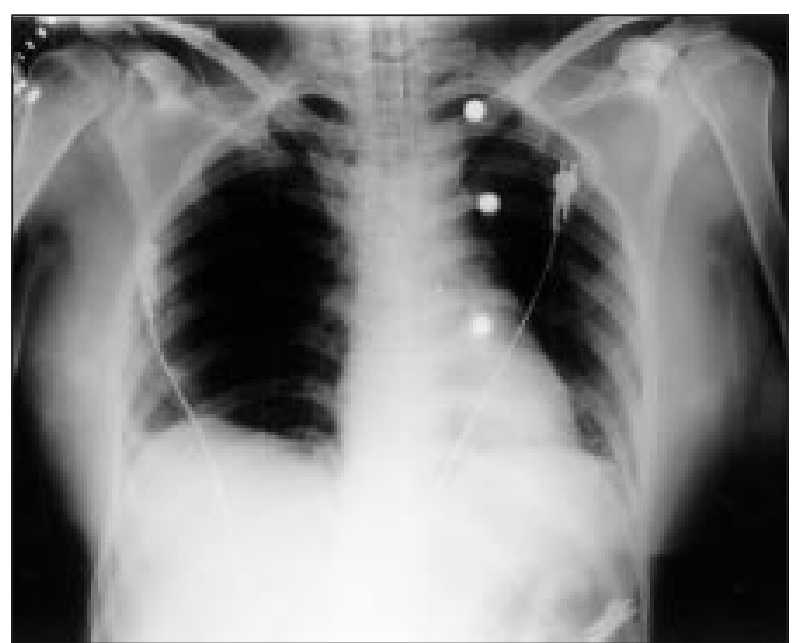

Fig. 10 - Radiografia de tórax em PA no pós-operatório com área cardíaca normal.

O ecocardiograma transesofágico permite meIhor avaliação da regurgitação mitral, bem como melhor visão do aneurisma, permitindo diagnóstico diferencial com defeito do septo interventricular ou pseudo-aneurisma de ventrículo esquerdo, muitas vezes sugerido pelo estudo hemodinâmico com ventriculografia ${ }^{(8)}$. Realizado no per-operatório, o ecocardiograma transesofágico nos dá segurança na avaliação da dinâmica da valva mitral, principalmente após plastia valvar, e da correção (exclusão) da dilatação aneurismática do átrio esquerdo.

Outro método diagnóstico não-invasivo que pode nos auxiliar é a tomografia computadorizada de tórax $(17,23)$, que demonstra a formação aneurismática e permite avaliar a integridade do pericárdio, afastando possíveis defeitos, muitas vezes, associados aos aneurismas da aurícula esquerda.

O estudo hemodinâmico é de grande importância permitindo:

- identificação da dilatação aneurismática em átrio esquerdo;

- análise da repercussão hemodinâmica através da manometria em câmaras direita e esquerda;

- avaliação funcional da valva mitral;

- detecção de disfunção ventricular;

- avaliação das artérias coronárias;

- pesquisa de lesões associadas.

Em alguns casos, o estudo hemodinâmico pode trazer dúvidas no diagnóstico, principalmente quando o átrio esquerdo cresce posteriormente ao ventrículo esquerdo. A cineangiografia pode sugerir a presença de pseudo-aneurisma de parede posterior do ventrículo esquerdo ou defeito do septo interventricular, sendo importante o diagnóstico diferencial (8).

Embora, freqüentemente, os aneurismas de átrio esquerdo sejam assintomáticos ${ }^{(15)}$, esta é uma lesão potencialmente fatal mesmo neste grupo de pacientes (24). Os acidentes tromboembólicos, as arritmias e a insuficiência cardíaca são as três principais complicações $(12,16,25-29)$. Não há relato na literatura de rotura de aneurismas de átrio esquerdo, embora haja a possibilidade de rotura, espontânea ou traumática (11). Em face das possíveis complicações, a opção terapêutica é o tratamento cirúrgico. A via de acesso pode ser a esternotomia mediana com estabelecimento de circulação extracorpórea ${ }^{(30)}$, ou a toracotomia esquerda sem circulação extracorpórea. Grandes aneurismas, sem colo definido, com trombos em seu interior, ou com lesões associadas geralmente necessitam de circulação extracorpórea. Pode-se realizar a ressecção aneurismática com reconstrução atrial ou a correção pelo interior do átrio esquerdo isolando o colo aneurismático e excluindo o aneurisma. Geralmente as arritmias, principalmente a fibrilação atrial, se presentes no pré-operatório, desaparecem no pós-operatório $(17,31,32)$ e o risco de fenômenos tromboembólicos tardios é pequeno (11).

\section{CONCLUSÕES}

Os aneurismas de átrio esquerdo não são comuns. Podem ocorrer ao nível da aurícula ou na parede livre do átrio esquerdo. O crescimento acentuado, posteriormente à parede posterior do ventrículo esquerdo pode levar ao diagnóstico de derrame pericárdico (pelo ecocardiograma), ou defeito do septo interventricular e pseudoaneurisma de ventrículo esquerdo (pelo cateterismo), impondo diagnóstico diferencial. O defeito no anel mitral posterior, bem como o crescimento aneurismático atrial pode levar ao desenvolvimento de insuficiência mitral. 0 tratamento cirúrgico é o de escolha, com possível preservação da valva mitral, e ressecção ou retirada do aneurisma. 0 risco cirúrgico é pequeno, e as possibilidades de prevenir ou tratar arritmias e ocorrência de fenômenos tromboembólicos são grandes. 
Bueno R M, Cerdeira Jr. M, Abensur H, Silva Jr. A, Calil O A, Ávila Neto V, Melo R F A - Defeito do anel fibroso mitral posterior com aneurisma de átrio esquerdo e insuficiência mitral: tratamento cirúrgico com sucesso. Rev Bras Cir Cardiovasc1999; 14 (4): 348-54.

RBCCV 44205-478

Bueno R M, Cerdeira Jr. M, Abensur H, Silva Jr. A, Calil O A, Ávila Neto V, Melo R F A - Defect of the posterior mitral fibrous ring with left atrial aneurysm and mitral insufficiency: successful surgical treatment. Rev Bras Cir Cardiovasc 1999; 14 (4): 348-54.

ABSTRACT: Atrial aneurysm is a rare condition and can be found on the atrial appendage or on the atrial wall. Most patients are asymptomatic, but arrhythmias, thromboembolism, and heart failure are common complications. We present a 39 years old female patient with posterior mitral ring defect causing a great aneurysm of the left posterior atrial wall with mitral insufficiency. The diagnosis was achieved by chest roentgenogram (marked prominence of the upper left heart border) and echocardiography (great left atrial aneurysm behind the left posterior ventricular wall with mitral insufficiency). Left ventricular cineangiogram suggested the presence of a false aneurysm of the left ventricle. The patient subsequently underwent surgical treatment with cardiopulmonary bypass. Posterior mitral valve annuloplasty was performed with aneurysm exclusion and posterior left atrial wall reconstruction using a bovine pericardial patch. The postoperative course was uneventful, and the patient was discharged sympton-free on the eighth postoperative day.

DESCRIPTORS: Heart aneurysm, congenital, surgery. Mitral valve insufficiency, surgery. Heart atrium. Heart valve, surgery.

\section{REFERÊNCIAS BIBLIOGRÁFICAS}

1 Semans J H \& Taussig H B - Congenital "aneurysmal" dilatation of the left auricle. Bull Johns Hopkins Hosp 1938; 63: 404.

2 Scardi S, Pandullo C, Nicolosi G L, Lutman M Aneurisma congenito dell'appendice atriale sinistra. G Ital Cardiol 1985; 15: 1098-100.

3 Morrow A G \& Behrendt D M - Congenital aneurysm (diverticulum) of the right atrium: clinical manifestations and results of operative treatment. Circulation 1968; 38: 124-8.

4 Hougen T J, Mulder D G, Gyepes M T, Moss A J Aneurysm of the left atrium. Am J Cardiol 1974; 33: 557-61.

5 Hansen J F, Rygg I, Efsen F - Intrapericardial left atrial aneurysm: report of a case and a review of the literature. Am Heart J 1974; 87: 113-6.

6 Ravikumar E, Kumar R, Chandy S T - Congenital intrapericardial aneurysm of the left atrium. Indian Heart J 1996; 48: 171-2.

7 Grinfeld R, Trainini J C, Roncoroni A, Fabrykant F, Cacheda $\mathrm{H}$, Tripodi $\mathrm{G}$ - Congenital aneurysm of the left atrium. Ann Thorac Surg 1985; 39: 469-71.

8 Reeves W C, Ciotola T, Babb J D, Buonocore E, Leaman $D$ - Prolapsed left atrium behind the left ventricular posterior wall: two dimensional echocardiographic and angiographic features. Am J Cardiol 1981; 47: 708-12.

9 De Sanctis R W, Dean D C, Bland E F - Extreme left atrial enlargement: some characteristic features. Circulation 1964; 29: 14-23.

10 Johnson J, Danielson G K, MacVaugh H 3d, Joyner C R-Plication of the giant left atrium at operation for severe mitral regurgitation. Surgery 1967; 61: 118-21.

11 Dusleag J, Klein W, Eber B, Gasser R, Weinrauch V, Rotman B - Noninvasive and invasive diagnosis of a huge congenital aneurysm of the left atrium: a case report. Angiology 1990; 41: 139-44.

12 Shaher R M, Alley R, Anis W, Mintzer J - Congenital enlargement of the left atrium. $J$ Thorac Cardiovasc Surg 1972; 63: 292-9.

13 Behrendt D M \& Aberdeen E - Congenital aneurysm of the left atrium. Ann Thorac Surg 1972; 13: 54-9.

14 Wang $T$, Anagnostopoulos C E, Resnekov L - Aneurysm of the body of the left atrium presenting with chest pain. Chest 1975; 67: 226-8.

15 LaBarre T R, Stamato N J, Hwang M H, Jacobs W R, Stephanides L, Scanlon P J - Left atrial appendage aneurysm with associated anomalous pulmonary venous drainage. Ann Heart J 1987; 114: 1243-5.

16 Krueger S K, Ferlic R M, Mooring P K - Left atrial appendage aneurysm: correlation of noninvasive with clinical and surgical findings: report of a case. Circulation 1975; 52: 732-8.

17 Brigui M, Ennabli K, Rémadi F, Belkhiria N, Chabrak S, Bouagina A - Importante dilatation aneurysmale de l'aurícule gauche. Arch Mal Coeur Vaiss 1994; 87: 1749-53.

18 Hirata T, Wolfe S B, Popp R L, Helmen C H, Feigenbaum $\mathrm{H}-$ Estimation of left atrial size using ultrasound. Am Heart J 1969; 78: 43-52.

19 Cate F J ten, Kloster F E, van Dorp W G, Meester G T, Relandt $\mathrm{J}$ - Dimensions and volumes of left atrium and ventricle determined by single beam echocardiography. Br Heart $J$ 1974; 36: 737-46. 
Bueno R M, Cerdeira Jr. M, Abensur H, Silva Jr. A, Calil O A, Ávila Neto V, Melo R F A - Defeito do anel fibroso mitral posterior com aneurisma de átrio esquerdo e insuficiência mitral: tratamento cirúrgico com sucesso. Rev Bras Cir Cardiovasc1999; $14(4): 348-54$.

20 Brown O R, Harrison D C, Popp R L - An improved method for echocardiographic detection of left atrial enlargement. Circulation 1974; 50: 58-64

21 Beppu S, Kawazoe K, Nimura Y et al. - Echocardiographic study of abnormal position and motion of the posterobasal wall of the left ventricle in cases of giant left atrium. Am J Cardiol 1982; 49: 467-72.

22 Ratshin R A, Smith M, Hood W P Jr. - Possible falsepositive diagnosis of pericardial effusion by echocardiography in presence of large left atrium. Chest 1974; 65: 112-4.

23 Root J D, Hammerman A M, Fischer K C - The CT appearance of the giant left atrium. Clin Imaging 1990; 14: $305-8$

24 Herbert W M, Arismendi L, Ruhstaller F D et al. Aneurysm of the left atrium associated with syncope and cyanosis. J Thorac Cardiovasc Surg 1965; 49: 535-9.

25 Hadowaki M H, Berger S, Shermeta D W, Arcilla R A, Karp R B - Congenital left atrial aneurysm in an infant. J Pediatr Surg 1989; 24: 306-8.
26 Coselli J S, Beall A C Jr, Ziaddi G M - Congenital intrapericardial aneurysmal dilatation of the left atrial appendage. Ann Thorac Surg 1985; 39: 466-7.

27 Moraes C R, Tompson C, Cavalcanti I et al. - Congenital aneurysm of the left atrium. Vasc Surg 1978; 12: 72.

28 Thadani V, Whitaker W, Watson D A - Congenital intrapericardial aneurysms of the left atrium. Thorax 1975; 30: 102-9.

29 Hall J \& Dobbs R H - Cerebral emboli from aneurysm of left atrial appendage. Proc $R$ Soc Med 1969; 62: 911.

30 Trehan N, Meharwal Z S, Sharma V K - Congenital left atrial aneurysm. Asian Cardiovasc Thorac Ann 1993; 4: $180-2$.

31 Lipkin D, Colli A, Somerville J - Aneurysmal dilatation of left atrial appendage diagnosed by cross sectional echocardiography and surgically removed. Br Heart $J$ 1985; 53: 69-71.

32 Sanderud A, Garman D, Hatle L, Rokseth R-Aneurysmal dilatation of the left auricle. Scand $J$ Thorac Cardiovasc Surg 1971; 5: 143-6. 